\title{
Integrating High Speed Navigation System to Avoid Accidents
}

\author{
S. Sandhya Rani ${ }^{1}$, B.V.K. Padamaja ${ }^{2}$, S. Gowri Lohitha ${ }^{3}$, T. Srinivas Rao ${ }^{4}$, V. Maraju ${ }^{5}$, K. Divya Sree \\ Students, Department of Electronics and Communication Engineering, D.M.S.S.V.H College of Engineering, \\ Machilipatnam, Andhra Pradesh 1,3,4,5,6
}

Assistant Professor, Department of Electronics and Communication Engineering, D.M.S.S.V.H College of

Engineering, Machilipatnam, Andhra Pradesh ${ }^{2}$

\begin{abstract}
This project mainly deals with the road accidents .It is designed to solve the accidents by using high speed navigation system. It will prevent the accidents happening while driving and it provide safety to passengers and pedestrians. To control these type of accidents automatic brake system is used. The distance of the object is measured by placing the Radar, Lidar and ultrasonic sensors in a car on both sides. The entire system is controlled by ARDUINO ATMEGA328. If any object approaches the car, then the sensors will alert and send the signal and then it gets reflected. Then the micro controller will send signals to the anti lock brake system .so that vehicle is controlled.
\end{abstract}

Keywords: Radar module, Lidar and ultrasonic sensor, high speed navigation system.

\section{INTRODUCTION}

Now a days the cars are designed with the monitoring system which are able to measure the distance of the two vehicles and by altering the person in the vehicle the occurrence of accidents is eliminated as early as possible[1]. To achieves such a system these sensors are prefers the ARDUINO ATMEGA328 will be used to perform the function accurately. Sensors are used to sense and monitor the system. Thus, this is high speed navigation and a safety system

PROBLEM: Due to the expansion of the vehicles the accidents are occurring frequently [1]. The number of road accidents have been surged road accidents are becoming one of the leading cause of deaths and disabilities across the world. Health statistics 2010 global report [2]

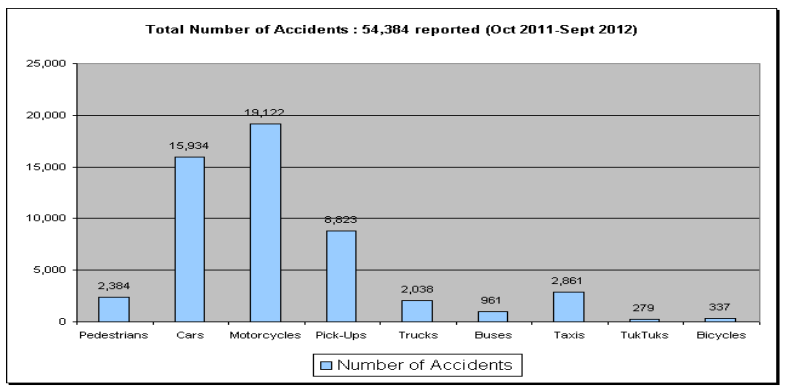

The major accidents are occurring by two wheeler and four wheelers during 2011 records there are 5 Lakhs road accidents (i.e.)[3] 1 road accident per minute and 1 road death for every 4 minutes .

Now, India is leading with china in road accidents. Thus this project is concentrating on safety measures while moving the vehicles.
The main concept of the project is based on "driverless car". [6] and to prevent the accidents by using high speed navigation system. The suitable sensors are ultrasonic sensor; Radar and Lidar are used for long range measurements.

\section{PROPOSED SYSTEM OVERVIEW}

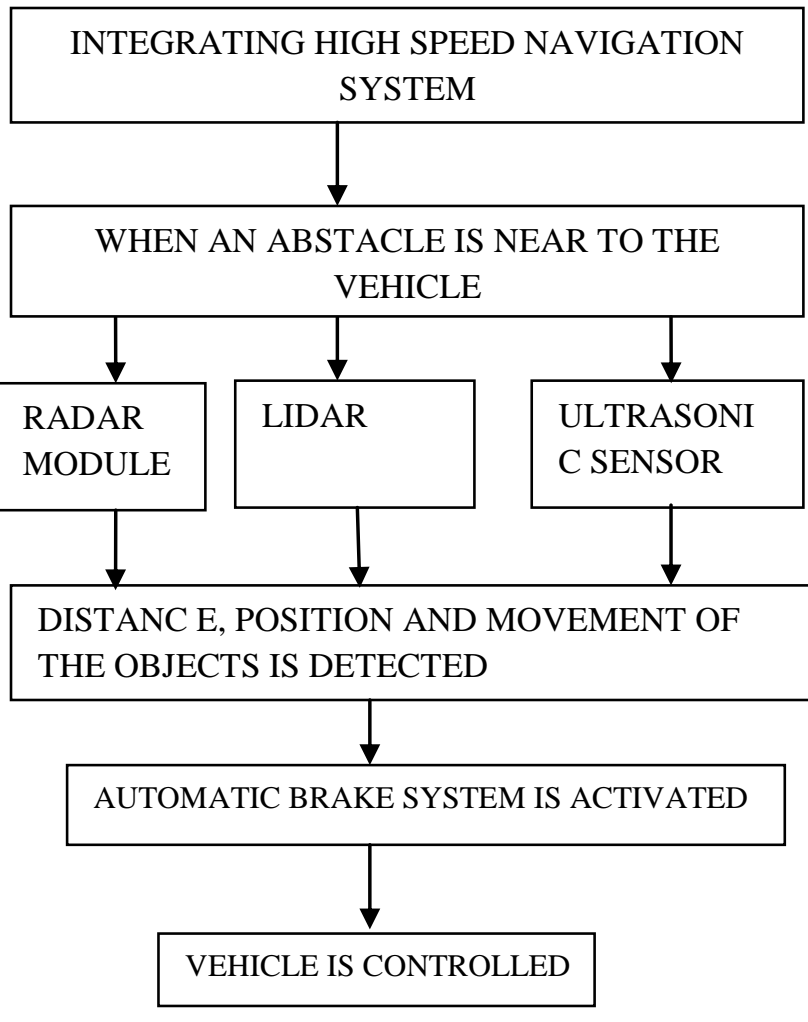


The navigation system is suggested system that contains radar, lidar and ultrasonic sensor by using this navigation we can navigate and estimate the position of the vehicle .It generates the steering commands for the vehicles to follow a specific path when there is an obstacle is in front of the vehicle then these sensors will sense and it gives the information to the brake system .Then automatic brake system is activated and the vehicle is controlled.

\section{RADAR:}

RADAR is abbreviated as "Radio detection and ranging". It is used to detect the movement and position of object. [4] The radar transmit the signal of radio waves and micro waves which bounce off any object in their path. Radar is an object detection system .It determines about direction , speed, altitude, range of the object.

RADAR uses Doppler effect to produce velocity and distance of object by bouncing a microwave signal of a desired target [4] and analysis the object motion has altered the frequency of the returned signal. [3]This gives accurate and direct measurement of target velocity.

HB100 Miniature Microwave Motion Sensor is a X-Band Bi-Static Doppler transceiver module. Its built-in Dielectric Resonator Oscillator (DRO) and a pair of Micro strip patch antenna array for motion detection. [5]

It can also be used for auto-door opening and vehicle speed measurement.

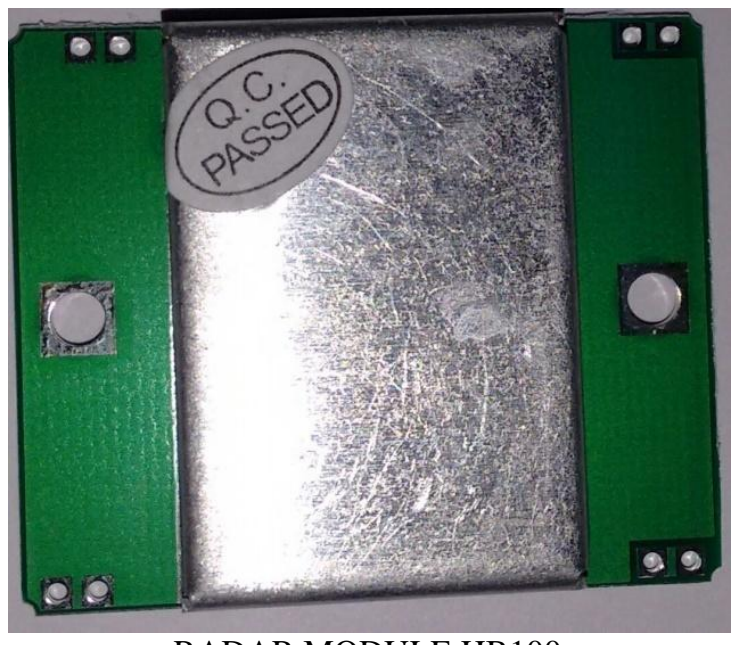

RADAR MODULE HB100

LIDAR:

LIDAR is abbreviated as "Light detection and Ranging" or "laser imaging detection and ranging". LIDAR itself send a pulse of near infrared waves and waits to return and scans from side to side covers larger area. Lidar is a fundamentally a distance measuring technology. It is a sensing method that uses light in the form of pulsed laser to measure ranges. Where a laser is fired from a transmitter and the reflected energy is captured it is a surveying method that measures distance to a target with laser light.
DC SERVO METER: Tiny and lightweight with high output power. Servo can rotate approximately 180 degrees (90 in each direction) and works just like the standard kinds but smaller.

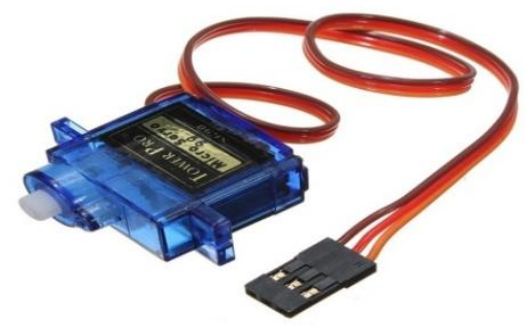

The servo motor is actually an assembly of four things: a normal DC motor, a gear reduction unit, a position-sensing device and a control circuit. The dc motor is connected with a gear mechanism which provides feedback to a position sensor which is mostly a potentiometer.

\section{ULTRASONIC SENSOR:}

Ultrasonic sensor actively transmit acoustic waves and receive them later. Ultrasonic transducer which transform an electrical signal into an ultrasonic wave and vice versa.[5] The ultrasonic sensor used to measure the distance of the object and to measure the amount of time taken by a pulse of sound to travel and return as echo.

Ultrasound signal carries the information about the variables to be measured. The ultrasonic transmitter and receiver is a pair it uses quartz crystal $40 \mathrm{khz}$ transducer is used [5].velocity of sound in air is $330 \mathrm{~m} / \mathrm{s}$. the ultrasonic sensor sends out a high frequency sound pulse and then times how long it takes for the echo of the sound to reflect back .

Sensor has 2 openings on its front:

1. Transmit ultrasonic waves (like a tiny speaker)

2. Receiver ultrasonic waves (like a tiny micro phone) In this project we are using Ultrasonic ranging module $\mathrm{HC}$ - SR04 provides $2 \mathrm{~cm}-400 \mathrm{~cm}$. The ranging accuracy can reach to $3 \mathrm{~mm}$. The modules includes ultrasonic transmitters, receiver and control circuit.

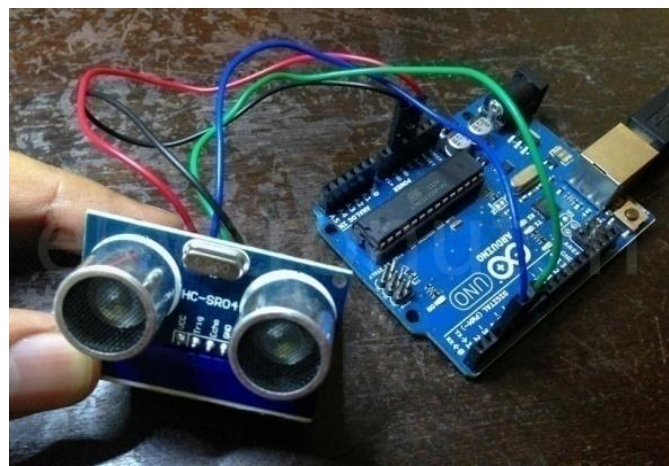

HC - SRO4 ULTRASONIC MODULE

The ultrasonic sensor has 4 terminals

1.Echo-output signal pin 4 


\section{International Journal of Innovative Research in}

\section{Electrical, Electronics, Instrumentation and Control Engineering}

\section{ISO 3297:2007 Certified}

Vol. 5, Issue 3, March 2017

\author{
2 .Trigger-input signal pin2 \\ 3. Vcc-1pin \\ 4. GND-3pin
}

\section{SYSTEM DESCRIPTION}

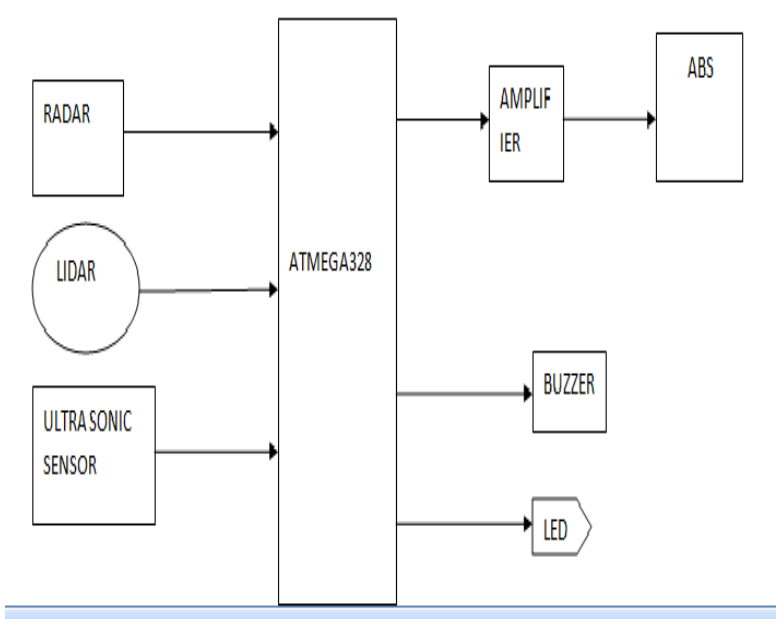

Block diagram of high speed navigation system
MICROCONTROLLER:

ATMEGA328 :The high-performance Microchip 8-bit AVR RISC-based microcontroller combines 32KB ISP flash memory with read-while-write capabilities[3]1KB EEPROM, 2KB SRAM, 23 general purpose I/O lines, 32 general purpose working registers, three flexible timer/counters with compare modes, internal and external interrupts, serial programmable USART, a byte-oriented 2-wire serial interface, SPI serial port, 6-channel 10-bit A/D converter , programmable watchdog timer with internal oscillator, and five software selectable power saving modes. The device operates between 1.8-5.5 volts. By executing powerful instructions in a single clock cycle, the device achieves throughputs approaching 1 MIPS per $\mathrm{MHz}$, balancing power consumption and processing speed.

\section{SOFTWARE:}

The ARDUINO UNO is a microcontroller board based on the ATmega328 (datasheet). It has 14 digital input/output pins (of which 6 can be used as PWM outputs), 6 analog inputs, a $16 \mathrm{MHz}$ crystal oscillator, a USB connection, a power jack, an ICSP header, and a reset button. It contains everything needed to support the microcontroller; simply connect it to a computer with a USB cable or power it with a AC-to-DC adapter or battery to get started. The Uno

This is the block diagram of the high speed navigation system which contains the RADAR,LIDAR and ULTRASONIC SENSOR The position and distance measuring modules and provides information to the microcontroller and then it sends the signals to the anti lock brake system so that the accidents are eliminated.

\section{OVERALL FLOW CHART:}

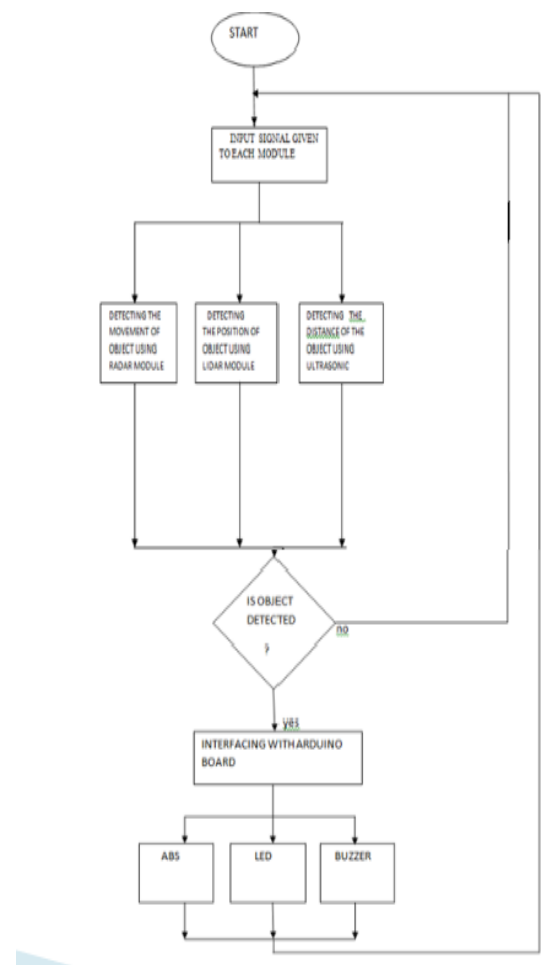

differs from all preceding boards in that it does not use the FTDI USB-to-serial driver chip [3]. The ARDUINO UNO can be powered via the USB connection or with an external power supply. The power Source is selected automatically

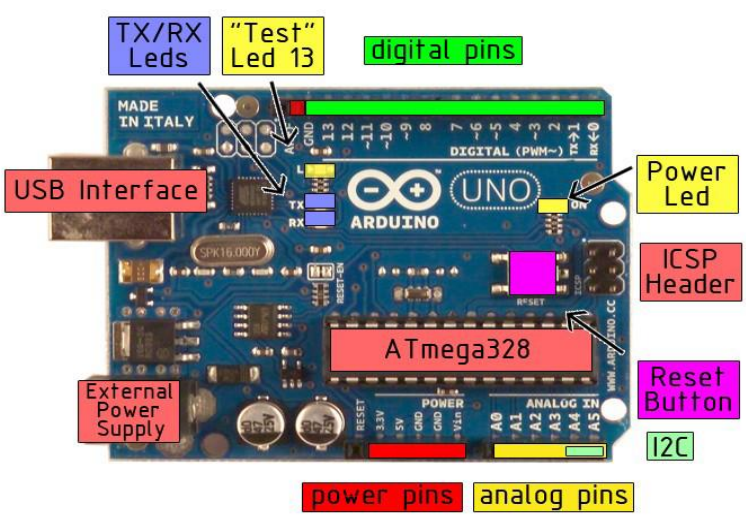

The ARDUINO UNO has a number of facilities for communicating with a computer, another ARDUINO, or other Microcontrollers. The ATmega328 provides UART TTL (5V) serial communication, which is available on digital pins $0(\mathrm{RX})$ and 1 (TX). An ATmega8U2 on the board channels this serial communication over USB and appears as a virtual com port to software on the computer. The ARDUINO software includes a serial monitor which allows simple textual data to be sent to and from the ARDUINO board. The RX and TX LEDs on the board will flash when data is being transmitted via the USB-to serial chip. 


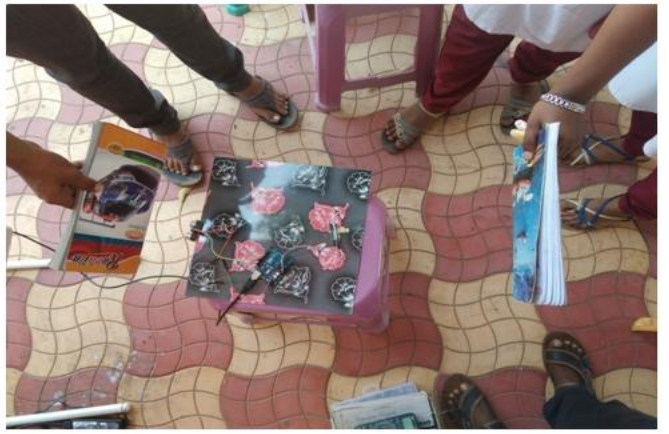

\section{RESULTS}

Fu11 range break
$46 \mathrm{~cm}$
Fu11 range break
$46 \mathrm{~cm}$
Fu11 range break
$45 \mathrm{~cm}$
Fu11 range break
$46 \mathrm{~cm}$
Fu11 range break
$46 \mathrm{~cm}$
Fu11 range break
$45 \mathrm{~cm}$
Fu11 range break
$46 \mathrm{~cm}$
Fu11 range break
$45 \mathrm{~cm}$
Fu11 range break
$46 \mathrm{~cm}$
Fu11 range break
$44 \mathrm{~cm}$
Fu11 range break
$44 \mathrm{~cm}$
Fu11 range break
$44 \mathrm{~cm}$
Fu11 range break
$44 \mathrm{~cm}$
Fu11 range break
$44 \mathrm{~cm}$
Fu11 range break
$44 \mathrm{~cm}$
Fu11 range break
$45 \mathrm{~cm}$

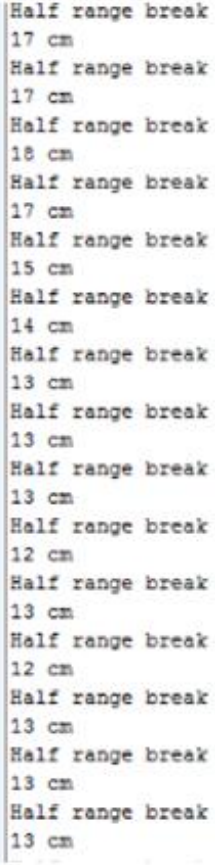

This project deals with how to prevent the accidents by using this integrating high speed navigation system .The sensors will sense and gives the accurate readings of the obstacle. The system is co-ordinated with these sensors so that it sends and receive the signals very fast and gives information to the brake system. Then the vehicle is controlled. This is more effective system and gives accurate results so that we can save lives of the people.

\section{REFERENCES}

[1] Venkatesh PL, Vivek C “"Safety Locking System of Car Door Using Sensors" .International Journal of Science and Research (IJSR) (2013): 6.14 |Volume 5 Issue 3, March 2016

[2] Faisal Muhaiyuddin , RahmatSanudin, Mohd Helmy Abd Wahab and Ayob Johari, "Development of Digital Vehicle Distance Monitoring system", (2009) IEEE, 612-616.

[3] T.K. Bhaumik, D. Sarkar "A Microcontroller Based Ultrasonic Range Finding Module for target detection from a Mobile Robot".

[4] K. Shrivastava, A. Verma, and S. P. Singh "Distance Measurement of an Object or Obstacle by Ultrasound Sensors using P89C51RD2" International Journal of Computer Theory and Engineering, Vol. 2, No. 1 February 2010.

[5] Ms C. Suganya , 2Ms A .Sivasankari “ Safety Car Drive by Using Ultrasonic And Radar Sensors" International Research Journal of Engineering and Technology (IRJET) Volume: 02 Issue: 04 | July2015. 1].

[6] Wikipedia/Driverless Car http//en.wikipedia.org/Driverless car.

\section{OUTPUT:}

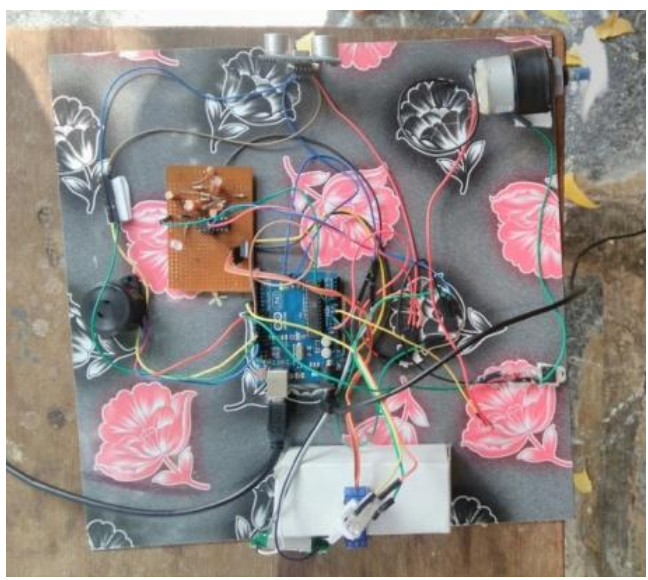

